\title{
Quantum Physics and ESP(An Epistemic Resolution)
}

\author{
John Nwanegbo-Ben \\ Department of Philosophy, Directorate of General Studies, Federal University of Technology, Owerri, Nigeria
}

Email address:

Johnjnb5005@gmail.com

\section{To cite this article:}

John Nwanegbo-Ben. Quantum Physics and Esp(An Epistemic Resolution). International Journal of Philosophy. Vol. 4, No. 3, 2016, pp. 11-17. doi: 10.11648/j.ijp.20160403.11

Received: April 15, 2016; Accepted: April 25, 2016; Published: May 26, 2016

\begin{abstract}
There is an epistemic relationship between Quantum physics and extra-sensory perception (ESP). The discovery and illumination gotten from the study of particle physics and the understanding of quantum physics brings to light a great resolution of the content of reality within the confines of the seen and unseen or from the mental and extra-mental. This paper posits that the inherent controversy in trying to comprehend the workings of "paranormal" acts such as telepathy and psychokinesis imbued by critics of paranormal phenomena can be resolved by an understanding of quantum physics and the uncertainty principle. The underlying position of this work is that everything in the universe, whether in the realm of the physical or the metaphysical are made up of energy and vibrations manifested in various forms. These forms include our bodies, minds, thoughts and in the elements. And uncertainties are parts and parcels of realities in both quantum mechanics and ESP.
\end{abstract}

Keywords: Quantum Physics, ESP, Sub-Atomic Particles, Uncertainty Principle, Telepathy, Psychokinesis, Magnetic Memory

\section{Introduction}

The advancement in contemporary physics has considerably improved such that roughly between 1924 and 1930 s, there was an entirely new theoretical approach to dynamics developed to account for sub-atomic behaviour. Quantum mechanics or wave mechanics was popularized with the works of Louis de Broglie who proposed that not only electromagnetic radiation but also that matter could have waves as well as particle aspect. The knowledge of this has great implication in understanding the workings of extra sensory perceptions especially on telepathy and psychokinesis. It is believed that there is really no difference between the radiation, wave or energy experienced by the works of experimental science and what we may call psychic science.

\subsection{An Understanding of the Origin of Quantum Physics}

Democritus of Abdera has been described by Erwin Schrödinger as one of the founders of Quantum mechanics. He was seen as the first quantum physicist not Max Plank as is traditionally known. He holds that all substances consisted of minute particles and that the different qualities of properties of substances were determined by different qualities of the atoms in them [1]. The earth from the position of Democritus and Leucippus emerged from the motion of atoms. Other atoms located outside the earth constitute what we know as the heavenly bodies. Man contained all conceivable types of atom and therefore "a microcosm of the universe" [2]. They went further to posit that properties of physical object like colour, smell, taste, solid, liquid and gaseous composition of the universe was explained by allotting different sizes and shapes to the atoms that constitute them.

In trying to explicate the paranormal, metaphysical or things that appear supernatural, he posited that the finest, most perfect, mobile and volatile of all atoms form the souls of animals and man. "It is hardly an exaggeration to say that the theory of Democritus remained in essentials unchanged until the $19^{\text {th }}$ century [3].

The philosophy of atomism is significant in trying to understand the essence of this paper. Atomism resurfaced in the $17^{\text {th }}$ century when Galileo revived it. He embraced atomism as a physical embodiment from the point of geometry. Newton's introduced the notion of forces, especially the gravitational force into Mechanics. Attractive 
forces between the atoms were new ideas and very helpful in understanding how solid and liquid hold together and how new compounds are formed from simpler ones. Robert Boyle promoted the idea that there are different types of atoms known as elements and that matter consists of particles of corpuscles in motion. After John Dalton who posits that atoms can neither be created nor destroyed, J. J. Thomson's research led to the discovery of the negatively charged electrons and the first idea of the structure of these indivisible atoms. With Ernest Rutherford's research it showed that some of the atoms consists of a closely packed core of positive charge particles known as the nucleus at the centre and surrounded by negatively charged electrons. He also observed that the energy associated with radioactive atoms is enormous. With prophetic insight Rutherford wrote in 1903 "There is no reason to assume that this enormous store of energy is possessed by radio elements alone. It seems probable that atomic energy in general is of a similar high order of magnitude [4].

Albert Einstein in the same model in the early part of the $20^{\text {th }}$ century gave a new conception of the atom in his quantum theory, thus denying the indestructibility of atom. Quantum theory has its major origin in the works of Max Planck, a theoretical physicist. He undertook an experiment in heat radiation and came to the conclusion that atoms never emits energy smoothly and continuously, but only intermittently in definite quantities or pulse. This "Pulse" of energy he calls "Quanta" of energy or photons.

Albert Einstein applied the same idea of Planck's quantum theory in his analysis of the photoelectric effect. It was already known that when light falls on a metal surface it ejects electrons, but this phenomenon cannot be explained by classical philosophers like Democritus. The conclusion is that both light (All electromagnetic radiation) and matter behave either as particles or as waves depending on how we observe them.

\subsection{What Quantum Physics Entails}

It may be necessary for us to understand what quantum physics entails, bearing in mind that we are not all physicists. A better understanding would give us the opportunity to juxtapose and demystify some acts of extra sensory perceptions within what we have labeled ordinarily as paranormal acts.

Quantum physics is the study or theory of how, what and why everything that makes up the universe as well as everything in it both within the confines of the seen and unseen are derived. It studies and tries to determine how everything in the cosmos came into being taking cognizance of its origin from the atomic and sub-atomic sphere. It is the study of the building blocks of the universe, an analysis of various forms of experiences and tracing theme back to molecules and energy. In fact quantum physics describes the universe as nothing more than vibrating strings of energy. This idea invariably posits that everything in the universe is made up of energy and vibration. This includes cells in our bodies and in the elements.

\subsection{The Metaphysics of Quantum Physics}

The idea of quantum physics is laden with metaphysical overtone and if we view issues bordering on the paranormal, we also see that they are metaphysical also because there appears to be no clear explanation of the claimed experiences of issues of ESP such as telepathy, psychokinesis, etc. the claims made by the experts in paranormal studies have been seen as uncertain because it cannot be quantified.

The uncertainty principle is the main theory in the physical science of quantum mechanics that explains the universe at atomic and sub-atomic levels. This principle emanated in order to explain how to measure the location of an electron around a nucleus. Werner Heisenberg in studying the works of Paul Dirac and Jordan discovered a problem with measurement of basic variables in the equations he was trying to solve. His analysis shows that uncertainties or imprecision always turned up if one tried to measure the position and the momentum of a particle at the same time. $\mathrm{He}$ concludes that these uncertainties or imprecision in the measurements are not the faults of the experimenter, but fundamental in nature inherent in quantum mechanics. In the eyes of the neophytes of nature and natural laws these uncertainties are supernatural, paranormal or metaphysical. Reasons for this possible conjecture is predicated on the fact that it is believed that natural sciences and its findings are not only empirically justifiable or quantifiable, but can also be predictable. In Quantum physics, the reverse appears to be the case because you cannot easily predict because the results are uncertain.

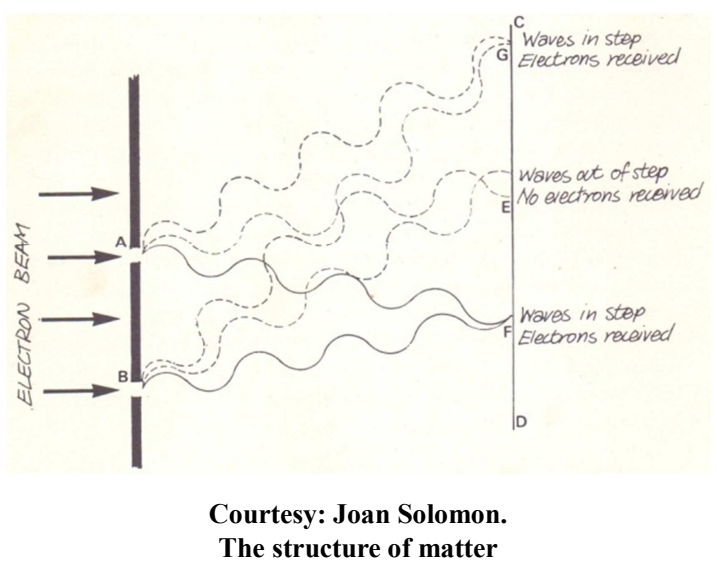

In juxtaposing religion, metaphysical experiences and paranormal acts as against empirical sciences, certain distinctive features are proposed. These features are that empirical scientific knowledge is accompanied with facts and their results are said to be certain. However, religion, or paranormal predictions or claims are laden with uncertainties and imprecision. The science of quantum physics exhibit uncertainties like that observed in religious circles. Its results are metaphysical because uncertainties or imprecision in measurements of variables are not the faults of the experimenter, they are beyond the scope of the scientist and cannot be predicted by any method or with any theoretical 
precise measurement. According to Paul levy, an enthusiast of the correlation of quantum physics and spirituality "Although Newtonian physics was and still is 'partially correct' from a strictly physical and finite material world view, quantum physics provides a much deeper, profound far more exiting and certainly more empowering understanding as to where all this 'physical stuff' (Matter is derived from" [5].

The uncertainty principle which is a result of quantum theory shows that at a given time it may be possible to measure the position of an electron with a very high degree of accuracy and it is possible to measure its momentum (and hence velocity) with a very high degree of accuracy; but beyond a certain limit, it is not possible to measure both together with a very high degree of accuracy. It is often thought that this result implies a rejection of determinism; that is the principle that every event is fully determined by antecedent causes [6]. The issue bordering on quantum physics appears to be beyond physics or can be said to be metaphysical.

\subsection{Extra-Sensory Perception (ESP) and Unknown Laws}

The knowledge of ESP may not be new among especially those who are critics or advocate of paranormal phenomena. However, we must confess that there are possibilities of those who are unenlightened on issues of extra-sensory perception. Our study and examples of ESP shall be limited to telepathy and psychokinesis.

By telepathy we mean the ability to become aware of another person's thought or feelings by means beyond the ordinary senses. The term was first used by an English Essayist and poet Fredric W. H. Myers in 1882, as "the communication of impressions of any kind from one mind to another independent of the recognized channels of sense [7]. Psychokinesis on the other hand is the ability to affect matter through a variety of mental force. Other terms that describe it include "remote mental influence, distant mental influences" or 'mind over matter'. Thus, it can be seen as "... the power of the mind to influence matter directly at a distance without any transfer of physical energy" [8]. Records show that psychokinetic feats have been performed by many individuals, but the force or energy behind it has been unknown.

We most times label extra-sensory perceptions paranormal. But the implication of the word paranormal may be misleading, for there is no reason to suppose that there is anything beyond the normal, in other words, outside the laws of nature about it, it is really normal and healthy people who seem to do best in these feats. According to Rosalind Heywood "The faculty is paranormal only in the sense that its cause is not perceptible to human senses or to their extensions, scientific instruments [9].

Extra sensory perception (ESP) which in general parlance may be called the sixth sense was used by a parapsychologist J. B. Rhine to describe the reception of knowledge or information not gained through the recognized physical senses but the mind. The mind has been described as a stream of consciousness and simply man at the sub-atomic level.
The mind has also been defined as a thinking thing. "As a man thinketh so he is". The mind is seen as the energy within man and is sometimes described as the soul. Democritus described the soul as a smote fine and volatile atom. While Aristotle goes on to affirm that the soul is a form or the "actuality of the body" [10]. The laws governing its workings are unknown that is why it falls within the confines of metaphysical discuss.

ESP activities occurs independent of the sense of sight, hearing or other sensory process. Individuals who have extra sensory perception to a high degree are said to be psychic. There is the conception that everybody has ESP; however others think that it is for special individuals with greater abilities. Experiments relating to extra sensory perception as expressed by Rosalind Heywood and from our personal experiences show that "Man is a creature who can make contact with distant events by an unknown process, which does not invoke the use of sign or hearing or touch, taste, smell and which to some extent at least is independent of time. [11].

The issue is that the simple understanding that such a faculty exist could lead to a revolution in thought about the nature of living creatures and their relation to their environment and the universe at large. The important point is that the laws which govern these phenomena are not yet known. The question of great philosophical or psychological interest is why is it not acclaimed a great discovery just like the uncertainly principle or the theory of relativity? The answer may be tied to the method of research which arose out of the debate over the issue of demarcating science from non-science or pseudo science.

\subsection{Dynamics of the Universe and Magnetic Memories}

The illumination gained through quantum physics exposes us to an understanding of what the extra-sensory perception might look like. This knowledge draws our interest to the conviction that the large scale structure and dynamics of the universe are intimately bound up with life on earth and the structure of atoms.

The Copenhagen interpretation of the issue of quantum physics reveals that energy is wave and at other experiment or interpretation of it, other probabilities occur. It shows that the thought and perceptions of the scientist doing the observation or experiment determined which one that manifest, either waves or particles. This implies that the subatomic particles (waves) being studied by the researcher respond to as particles (matter) based on the individual thought of the scientist who was studying it. By implication, the mode of thought or belief of the scientist or his perceptions determines the result of the research.

The belief by most scientists that life on earth started with a single organism is predicated on the conception that we all have genetic connections of different degrees through this single organism. This may be likened to the monads of Leibnitz. The Greek word Monad means "Unity". Everything is made up of infinite number of monads. He however, denied the reality of matter. One monad always 
predominates. In the case of human beings the dominant monad is mind. There must be some relation between all the monads which make up the universe [12]. Man has genetic link with one another like a telephone exchange and this is possible because of what we have come to understand as magnetic memory from one person to the other. By this, we opined that all human life share a common branch point, this branch point is a long way in the past.

Magnetic Memory is the memory and link due to the storage of data on a magnetized medium. It could be through genetics and wave properties of matter and mind. This medium is conjectured as responsible for the interconnectedness of things in the universe.

The interconnectedness of man through magnetic memories may account for the possibility of telepathy, psychokinesis and other psychic phenomena. This is because our thought and being can be transmuted as particles of matter or in form of wave.

Quantum physics and the theory of magnetic memories express that the whole of the universe or the entire cosmos is one, which is energy. Our physical body and thought forms are simply energies from different levels.

\subsection{Magnetic Memories and ESP}

In this segment we hope to identify experiences observed in ESP and see its correlation to magnetic memories. Our interest in given examples of some of these extra sensory perceptions are due to the fact that we at various points in our lives have direct experiences of these phenomena. The illumination given by our knowledge of particle physics only helps us to understand why the assumed paranormal should be seen as normal or the perceived supernatural as natural.

The science of telepathy and psychokinesis as a case study is perceived to be possible due to the activity of magnetic memory. This magnetic memory is responsible for the interconnectedness of thought waves or particles.

There are multiple of cases of interconnectedness between men that makes it possible for us to believe that telepathic and psychokinetic rapour is a fact. For example, twins having some type of pain together, giving birth together or dying together. There was a special issue published in an Australian Magazine - Truth, as recounted by Percy Seymour “... twin sisters Helen and Peg, one night three quarters of an hour before midnight, Peg was killed in a car crash when the steering wheel penetrated her chest. At the same time Helen woke up screaming saying, she has a severe pain in her chest, on her way to the hospital, she died in the ambulance" [13]. This shows that there is interconnectedness between Helen and Peg despite their distance from one another. There is also a mysterious synchronism between twins that justify interconnectedness due to magnetic memory. A particular example recorded by Seymour is between identical twins. It occurred at 4.35 am on a Saturday in July 1948. At this time, Alice Lamba was reading in the palour of her home in Springfield, Illinois, U.S.A. when she suddenly felt a jolt on the left side of her body. This was followed by a feeling of shock and sharp stabbing pain in her side. She ten seems to be knocked off her chair by some unseen force and just before fainting, she said "Something has happened to Dianne" At that very instant, seventy miles away, Dianne her twin was travelling on a train that had just been derailed, and she had been thrown across the carriage. When she woke up in hospital, it was to find that she had suffered severe concussion and had fractured two ribs. Alice also complained of pains in her side, and when she was X-rayed it turned out that she had fractures just like her sister at the same two ribs [14].

The author most times had telepathic experiences that amazed his friends, this occurs when he reveals the thoughts of these friends during conversations. They sometimes shiver and ask if he was a prophet. What actually took place was transference of thought waves or radiation from their minds to his mind because they were naturally interconnected and his mind was receptive.

Many twins have telepathic link just as some individuals that are highly telepathic. There are many examples within our traditional communities that justify the issues of extrasensory activities. What we wish to stress is that these activities are as natural as the laws in physics.

Current research hypothesizes that every part of the bodymental, physical and emotional forms a continuous interconnected bio-energetic communication network. Like the uncertainty principle which we observe has reflected the preconceived idea of the scientists during experiment or research, health and ill health appear to reflect the mind set of individuals. There was a case recounted by J. O. Mume, a woman who gave birth to a male child and became mentally deranged. In addition to her mental problem were malaria fever, cold and headache. During medication on these symptoms, her constant complain was "I have a snake going up and down in my stomach trying to take my life". She wept and asked her doctor, "Doctor, can you remove this snake from my belly?"

The doctor being conscious of the inherent powers of the mind in relation to belief and how it affects matter had to exploit his knowledge of psychology. He sent one of his assistants to get a snake dead or alive, this was done. He gave the patient herbal emetic to induce vomit. While she vomited, he smeared pepper into her eyes in order to blindfold her from observing his tricks. At this instance, he brought out the dead snake he concealed in a bottle and placed it among her vomit and asked her to open her eyes, only for her to see a dead snake in front of her. The doctor in addition, instantly shouted in an unusual voice "your trouble is now over, I have killed the snake that is going up and down in your stomach, and I have made you to vomit it [15].

With this technique, the woman's insanity disappeared and she was happy as she pointed at the snake telling her husband "I told you that there was a snake in my body".

The lady was mentally ill because she had a belief that a snake was right inside her. Then she was tricked to believe again that the snake had been killed and she became well and sound. This shows that probably there is a clear interconnectedness between the mental and physical and that words have special effects like sound waves with certain 
vibration that affects the higher dimension of the physical human being.

Mume's experiments may have been successful as a psychological remedy or relief but we do not know if the healing was sustained or mere momentary relief. The question; however is if she gets to know that she was tricked, would she be able to contain the news and remain mentally sound? If not so, then it is the fact that the uncertainty principal and ESP phenomena are controlled by our thoughts.

\subsection{Human Intensions \& Psychokinesis}

An interesting area of ESP or psychic science is that of psychokinesis. Psychokinesis is the ability to affect matter through the activity of the mind. It is a term used to describe "ability of the mind to influence matter, time, space or energy without the use of any currently known type of physical means" An example of psychokinesis includes distorting or moving an object [16].

Records show that psychokinetic feats have been performed by many individuals, but the force or energy behind it has been unknown. Notable claimants of psychokinetic abilities are Uri Geller, an Israeli, famous for his spoon-bending demonstrations. Nina Kulagina an alleged Soviet psychic of the late 1960s and early 1970s. Another is Felicia Parise, an American Medical Laboratory Technician who allegedly was able to repeatedly demonstrate psychokinetic movement of small objects beginning in the 1970s. In the first reported instance, it was spontaneous and then with practice by intense conscious intention she said, her inspiration for making the attempt was in viewing the blackand-white films of Nina Kulagina performing similar feat". [17] Another individual who developed this ability by viewing the films of Nina was Allan Vinogradovo of the Soviet Union who is said to have been tested successfully under controlled laboratory experiment.

Number of researchers has conducted studies to determine whether mere human intention could affect the movement of moving object or affect matter directly. The author has watched with amazement unequal legs of a young lady due to a strange ailment and a man's left hand shorten by an accident all brought and made equal by an intense conscious intension of a great healer in Warri, Nigeria on the $4^{\text {th }}$ of March, 2016. Within few hours of the last feat, the same healer, with intense conscious intention was able to reverse positively a police officer's tongue protruded out of his mouth for two years without solution.

Extensive research has been conducted by John Hasted at the University of London's Birkbeck College. He hypothesized that an unknown form of conduction of electrical charge from the subjects bodies through the atmosphere to the sensors of his equipment [18] Other experiments to this are that conscious intention can influence the growth and movement of biological studies. This was conducted by Dr. Carol B. Mash in which bacterial growth was psychokinetically accelerated and retarded according to the intentions of random selected college students.

These researches raise many questions regarding the nature of energy or powers involved that appears to transcend experimental scientific explanation. Another penitent question could be, does quantum physics adequately explain ESP?

\section{Uncertainties in Quantum Physics and ESP}

The question we need to ask is does the knowledge of quantum physics explain issues that involve Extra Sensory Perception? We have through the knowledge of particle physics encountered the notion of the uncertainty principle, a situation where imprecision always turned up if one tried to measure the position and the momentum of a particle at the same time. The conclusion made was that uncertainties and imprecision in the measurements are not the faults of the experimenter, but fundamental in nature inherent in quantum mechanics.

How does this relate to ESP? The results of Extra-Sensory Perception are uncertain bearing in mind its method. The similarity within the understanding of quantum mechanics, the uncertainty principle with extra-sensory perception cannot be farfetched. The reason is that uncertainties border on each experiment. The researchers may not accurately determine inductively that today's results would be as accurate as that of yesterday because imprecision always turned up. Research by John Hasted on ESP at the University of London's Birbeck College hypothesized that an unknown form of conduction of electrical charge was observed through the atmosphere from the bodies of the subject. The statement "unknown form of conduction" indicates similarity with uncertainty in procedures and results.

Albert Einstein's E $=\mathrm{Mc}^{2}$ proved that all things broken down to their most basic form consist of the same stuff. What is this stuff-Pure energy? Energy makes up all things. Without a basic understanding of quantum physics, we may think that there are different energies such as energy that powers our homes, which make us move about, fuels our cars.

The cosmos from the foregoing is just a form of universal interconnected energy which obeys the science of physics based on the principles of everything is energy. And energy follows thought as expressed in the uncertainty principle and extra sensory perception and that whatever man focuses on, he gets more of it. Our thoughts and feelings whether in the process of research or not send out magnetic field, and universal energy matches our alteration by giving us more of the same resonant energy. Just as the sub atomic particles took whatever form the scientist studying them, expected them to take based on their thoughts, events, conditions and circumstances that make up our reality take shape as we expect and believe they will. Intense conscious intention observed in faith healing and psychokinesisis is an expressions of energy in motion from one form to the other.

Quantum Physics tells us that it is the act of observing an object (events, conditions and circumstances) that causes it to 
be there and the outcome is based only on how we observe it.

Quantum physics appears to reconcile science and religion. It tends to say "As a man thinketh, so is he". If our thoughts can be telepathically transferred and psychokinetically move an object by intention, it implies that if we think negatively or have negative belief system about ourselves, we will manifest illness in our bodies. Thus, thought is energy. As we "believe so shall we receive. Within the religious parlance, we can say "Faith (positive thought energy) is the substance of things hoped for, the evidence of things not seen". Whatsoever ye desire when ye pray (thought in motion) believed that ye receive and it shall be given to you". If we can conceive and believe in the materialization of our hopes, dreams and desires, they already exist as a wave in the quantum field (which is infinite in nature) as a probability of existence only awaiting you to make them real.

\section{Conclusion}

This research concludes, bearing in mind that our knowledge of Quantum Physics and uncertainty principle exposes that Extra-Sensory Perception (ESP) are due to natural laws expressed in better terms as the universe vibrating in strings of energy. That an understanding of this, will position us to know that our thoughts are not only energy, but exist as a wave in the quantum field as a probability of existence that can be controlled or channeled to accomplish a desire in the universe. It posits that the cosmos is a universal interconnected energy which obeys the law of physics and made earlier by inherent magnetic memories in the universe.

This paper does not classify ESP phenomena as religious experiences with the conception that our belief might be replicated as it is backed by faith, but that the content of religious faith and belief expresses the theory of quantum physics and uncertainty principle. Our study here sees ESP as the melting point of science and religion.

\section{References}

[1] V. Smilga, In the Search for Beauty. Moscow: Mir Publishers. 1970. Pp. 19-20.

[2] Stephen F. Mason A history of Science. New York: Collier Books. 1970. Pp. 33-34.

[3] W. K. C. Guthrie The Greek Philosophers: from Thales to Aristotle. London: Methuen \&Company Ltd. 1976. P. 57.

[4] Joan Solomon. The Structure of Matter. England: David \& Charles. 1973. P. 97.

[5] Paul Levy, Quantum Physics. www.awakeninthedream.com /quantum-physics-the. Retrieved Now 2015

[6] Thomas Meitner (ed) The Penguin Dictionary of Philosophy. London: Penguin Books. 2000. P. 578.

[7] W. A. Redmond Telepathy. Microsoft Student. DVD Microsoft Co-operation, 2008.
[8] Rosalind Heywood. Beyond the Preach of Sense: An Inquiry into Extra Sensory Perception. New York: E. P. Dutton and Company, Inc. 1974. P. 162.

[9] Ibid, P. 15.

[10] Aristotle, On the Soul, Book 2. 412. a 15-28.

[11] Rosalind Heywood. Opcit P. 10

[12] Samuel Enoch Stumpf. Philosophy: History \&Problems. New York: Mc Graw-Hill Inc. 1989. P. 256.

[13] Percy Seymour. The Paranormal: Beyond Sensory Science. London: Arkana Penguin Books. 1992. P. 132.

[14] Ibid. p. 130

[15] J. O. Mume "How I Acquired the knowledge of traditional medicine" in Traditional Healing: New Science or New Colonialism (Essay in critique of medical Anthropology). Philip Singer (ed) London: Couch Magazine Ltd. 1977. Pp. 153-156.

[16] Farlex Partner, Medical Dictionary: Psychakinesis. $\mathrm{http} / /$ medical-dictionary. The free dictionary. copsychokinesis. Retrieved July 16, 2014.

[17] Mind over Matter (Volume of Mysteries of the Unknown Encyclopedia Series). New York: Time-Life Book. 1988. P. 27.

[18] The Roots of Consciousness: science, Psychokinesis. www.williamjanes.com/science/pk.htm

[19] Armstrong, D. M. A Materialistic Theory of the Mind. London: Rutledge and Kegan Paul. 1968.

[20] Aristotle, On the Soul. Book 2. 412.

[21] Baker, R. Human Navigation and the Sixth Sense. Machester University Press. 1988.

[22] Barley, Cyril, The Greek Atomists and Epicurus. New York: Russel and Russel Inc. 1964.

[23] Bell, John S. Speakable and Unspeakable in Quantum Mechanics. Cambridge University Press. 1988.

[24] Broughton, Richard S. Parapsychology: The Controversial Science London: Ballantine Books. 1991.

[25] Berger, Arthur, S.; Berger, Joyce. The Encyclopedia of Parapsychological \& Psychical Research. New York: Paragon House. 1991.

[26] Capra F., The Tao of Physics, New York: Fontara Press. 983

[27] Davies, P. W. C. Superstrings: A Theory for Everything. Cambridge University Press. 1988.

[28] Frazer, J. G. The Golden Bough: A Study in Magic and Religion. Abridged Edition, London: Macmillan \& company. 1963.

[29] Guiley, Rosemary Ellen, Encyclopedia of the Strange, Mystical and Unexplained. New York: Grammercy Book. 1991.

[30] Guthrie, W. K. The Greek Philosophers: From Thales to Aristotle. London: Methuen \& Company Ltd. 1976.

[31] Heywood, Rosalind, Beyond the Reach of Sense. New York EP, Dutton and Co. Inc. 1974. 
[32] Inglis, Brain. The Paranormal: An Encyclopedia of Psychic Phenomena. London: Paladin. 1986.

[33] Mume J. O. "How I Acquired the Knowledge of traditional Medicine" in Traditional Healing New Science or New Colonialism (Essays in Critique of Medical Anthropology) Philip Singer (ed). London: Conch Magazine Ltd. 1977.

[34] Mason, F. Stephen A History of the Sciences; New York: Collier Books, 1979.

[35] Milton, Richard Forbidden Science: Suppressed Research that Could Change our Lives; London: Fourth Estate. 1994.
[36] Solomon, Joan The Structure of Matter: The Growth of Man's Idea on the Nature of Matter. Newton Abbot Devon David \& Charles. 1973.

[37] Seymour, Percy The Paranormal: Beyond Sensory Science, London: Arkana Penguin Books 1992.

[38] Seymour, Percy Cosmic Magnetism London \& Adam Hilger, 1986.

[39] Smith, C. \& Best S. Electromagnetic Man. Dento 1989.

[40] Talbot, M. Beyond the Quantum; New York, Bantam Books, 1988. 